\title{
Communication
}

\section{Mice Immunized with the Vaccine Candidate HexaPro Spike Produce Neutralizing Antibodies against SARS-CoV-2}

\author{
Chotiwat Seephetdee ${ }^{1}\left(\mathbb{D}\right.$, Nattawut Buasri ${ }^{1}$, Kanit Bhukhai ${ }^{2}$, Kitima Srisanga ${ }^{1}$, Suwimon Manopwisedjaroen ${ }^{3}$, \\ Sarat Lertjintanakit ${ }^{1}$, Nut Phueakphud ${ }^{1}$, Chatbenja Pakiranay ${ }^{1}$, Niwat Kangwanrangsan ${ }^{4}$, \\ Sirawat Srichatrapimuk ${ }^{5}$, Suppachok Kirdlarp ${ }^{5}$, Somnuek Sungkanuparph ${ }^{5}$, Somchai Chutipongtanate ${ }^{5,6,7}$ (D), \\ Arunee Thitithanyanont ${ }^{3} \mathbb{D}$, Suradej Hongeng ${ }^{6}$ and Patompon Wongtrakoongate $1,8, * \mathbb{D}$
}

Citation: Seephetdee, C.; Buasri, N.; Bhukhai, K.; Srisanga, K.;

Manopwisedjaroen, S.; Lertjintanakit,

S.; Phueakphud, N.; Pakiranay, C.;

Kangwanrangsan, N.;

Srichatrapimuk, S.; et al. Mice

Immunized with the Vaccine

Candidate HexaPro Spike Produce

Neutralizing Antibodies against

SARS-CoV-2. Vaccines 2021, 9, 498.

https://doi.org/10.3390/

vaccines 9050498

Academic Editors:

Luis Martinez-Sobrido,

Soo-Hong Lee, Hansoo Park,

Jagathesh Chandra Rajendran and

Jaganathan K. Semburakkiannan

Received: 2 March 2021

Accepted: 5 May 2021

Published: 12 May 2021

Publisher's Note: MDPI stays neutra with regard to jurisdictional claims in published maps and institutional affiliations.

Copyright: (c) 2021 by the authors. Licensee MDPI, Basel, Switzerland. This article is an open access article distributed under the terms and conditions of the Creative Commons Attribution (CC BY) license (https:/ creativecommons.org/licenses/by/ $4.0 /)$
1 Department of Biochemistry, Faculty of Science, Mahidol University, Bangkok 10400, Thailand; chotiwat.see@student.mahidol.ac.th (C.S.); nattawut.bua@alumni.mahidol.ac.th (N.B.); kitima.sri@mahidol.ac.th (K.S.); sarat.lej@student.mahidol.ac.th (S.L.); nut.phe@alumni.mahidol.ac.th (N.P.); chatbenja.pai@student.mahidol.ac.th (C.P.)

2 Department of Physiology, Faculty of Science, Mahidol University, Bangkok 10400, Thailand; kanit.bhu@mahidol.ac.th

3 Department of Microbiology, Faculty of Science, Mahidol University, Bangkok 10400, Thailand; swiboonut@gmail.com (S.M.); arunee.thi@mahidol.edu (A.T.)

4 Department of Pathobiology, Faculty of Science, Mahidol University, Bangkok 10400, Thailand; niwat.kan@mahidol.ac.th

5 Chakri Naruebodindra Medical Institute, Faculty of Medicine Ramathibodi Hospital, Mahidol University, Samut Prakan 10540, Thailand; sirawat.sri@mahidol.ac.th (S.S.); suppachok.kir@mahidol.ac.th (S.K.); somnuek.sun@mahidol.ac.th (S.S.); schuti.rama@gmail.com (S.C.)

6 Department of Pediatrics, Faculty of Medicine Ramathibodi Hospital, Mahidol University, Bangkok 10400, Thailand; suradej.hon@mahidol.ac.th

7 Department of Clinical Epidemiology and Biostatistics, Faculty of Medicine Ramathibodi Hospital, Mahidol University, Bangkok 10400, Thailand

8 Center for Neuroscience, Faculty of Science, Mahidol University, Bangkok 10400, Thailand

* Correspondence: patompon.won@mahidol.ac.th

Abstract: Updated and revised versions of COVID-19 vaccines are vital due to genetic variations of the SARS-CoV-2 spike antigen. Furthermore, vaccines that are safe, cost-effective, and logistic-friendly are critically needed for global equity, especially for middle- to low-income countries. Recombinant protein-based subunit vaccines against SARS-CoV-2 have been reported using the receptor-binding domain (RBD) and the prefusion spike trimers (S-2P). Recently, a new version of prefusion spike trimers, named HexaPro, has been shown to possess two RBD in the "up" conformation, due to its physical property, as opposed to just one exposed RBD found in S-2P. Importantly, this HexaPro spike antigen is more stable than S-2P, raising its feasibility for global logistics and supply chain. Here, we report that the spike protein HexaPro offers a promising candidate for the SARS-CoV-2 vaccine. Mice immunized by the recombinant HexaPro adjuvanted with aluminum hydroxide using a prime-boost regimen produced high-titer neutralizing antibodies for up to 56 days after initial immunization against live SARS-CoV-2 infection. Also, the level of neutralization activity is comparable to that of convalescence sera. Our results indicate that the HexaPro subunit vaccine confers neutralization activity in sera collected from mice receiving the prime-boost regimen.

Keywords: HexaPro; spike; SARS-CoV-2; vaccine

\section{Introduction}

The coronavirus disease 2019 (COVID-19), caused by the novel coronavirus, severe acute respiratory syndrome coronavirus 2 (SARS-CoV-2), is a current global pandemic. The incidence for this pandemic reported by World Health Organization (WHO) on 31 March 2021, has included 130 million cumulative confirmed cases and over 2.84 million deaths globally. There is an urgent need for preventative vaccines and therapeutics. SARS-CoV-2 
is an enveloped, single-stranded RNA virus. Its genome encodes four structural proteins comprising of the spike (S), membrane glycoprotein (M), envelope (E), and nucleocapsid $(\mathrm{N})$ proteins. The spike protein mediates viral entry by binding to the host receptor angiotensin-converting enzyme 2 (ACE2) via the receptor-binding domain (RBD). This interaction triggers a substantial conformational alteration of the spike from a prefusion conformation to a highly stable postfusion conformation [1-4]. The spike protein can induce the production of neutralizing antibodies in patients, indicating its immunogenic property. Thus, it has been widely adopted for vaccine development. However, the ongoing COVID-19 pandemic has led to SARS-CoV-2 spike variants with serious concerns such as D614G, N501Y, E484K, and 69/70 deletion [5-7]. Some of these variants can be highly transmissible and capable of escape vaccine-induced neutralizing antibody responses [8].

A key strategy for vaccine design against coronaviruses SARS-CoV and MERS-CoV has aimed at stabilizing the metastable prefusion conformation of the spike protein homologs $[9,10]$. The prefusion stabilization has been achieved with two consecutive proline substitutions (S-2P) in a turn between the central helix and heptad repeat 1 (HR1). These $\mathrm{S}-2 \mathrm{P}$ variants, together with a C-terminus foldon trimerization domain, have been shown as a superior immunogen [10]. As a consequence, the SARS-CoV-2 S-2P has been employed in currently used vaccines including mRNA-1273 [11], BNT162b2 [12], and ChAdOx1 [13].

In this work, we aim to provide a proof-of-concept of a recently published prefusionstabilized spike ectodomain, namely HexaPro, developed by McLellan and colleagues [14] as a potential COVID-19 subunit vaccine. We show that the HexaPro subunit vaccine administered with aluminum hydroxide adjuvant in mice elicits a strong neutralizing antibody response against SARS-CoV-2. This finding holds a promise towards a nextgeneration coronavirus vaccine development using the HexaPro spike protein.

\section{Materials and Methods}

\subsection{Ethics Statement}

Mouse experiments were performed under the Animal Ethics approved by Faculty of Science, Mahidol University (MUSC63-016-524). PCR-confirmed COVID-19 patients $(n=58)$ were hospitalized at Chakri Naruebodindra Medical Institute, Faculty of Medicine Ramathibodi Hospital, Mahidol University. Serum specimens were collected from patients 14-30 days post-infection. The study protocol and human ethics were approved by Faculty of Medicine Ramathibodi Hospital (COA. MURA2020/568).

\subsection{Expression and Purification of HexaPro Subunit Vaccine}

The mammalian expression plasmid containing SARS-CoV-2 HexaPro spike was obtained from Addgene (Addgene plasmid \# 154754; http:/ /n2t.net/addgene:154754; RRID: Addgene_154754; accessed on 1 December 2020). HEK293T cells were transiently transfected with the HexaPro plasmid by calcium phosphate transfection. Cells and culture medium were separated by centrifugation. The supernatant was concentrated with Amicon ${ }^{\circledR}$ Ultra-15 Ultrace-30K centrifugal filter unit (MERCK). Cell protein contents were extracted with a lysis buffer composed of $50 \mathrm{mM}$ sodium phosphate, $300 \mathrm{mM} \mathrm{NaCl}$, $20 \mathrm{mM}$ imidazole, 1X CompleteTM EDTA-free protease inhibitor cocktail (Roche), $1 \mathrm{mM}$ Phenylmethylsulfonyl fluoride (PMSF, Sigma-Aldrich, St. Louis, MO, USA), and 1\% Triton-X (Sigma-Aldrich). Protein extracts were filtered through a $0.22 \mu \mathrm{m}$ NalgeneTM syringe filter (Thermo ScientificTM). S HexaPro protein was then purified with HisTrap $\mathrm{HP}$ (cytiva) equilibrated with a buffer composed of $50 \mathrm{mM}$ Sodium phosphate, $300 \mathrm{mM}$ $\mathrm{NaCl}$, and $20 \mathrm{mM}$ imidazole. Fractions containing HexaPro were pooled and exchanged to phosphate-buffered saline (PBS). Purified protein was digested with HRV3C protease to remove purification tags. The protein was further purified with Sephacryl S-300 HR (GE Healthcare, Chicago, IL, USA) with PBS. Fractions that contained HexaPro protein were pooled and analyzed with SDS-PAGE and Western blot against the SARS-CoV-2 RBD protein (Sino Biological, Cat\#40592-T62) or pooled convalescent sera. The purified protein was kept at $-80^{\circ} \mathrm{C}$ until use. 


\subsection{Immunofluorescence Staining}

HeLa cells were transiently transfected with the plasmid encoding HexaPro using lipofectamine 3000 (Invitrogen, Cat\#L3000008, Carlsbad, CA, USA). Cells were fixed with $4 \%$ PFA and were incubated with either a polyclonal antibody against the SARS-CoV-2 RBD protein (Sino Biological, Cat\#40592-T62) or a monoclonal antibody against the SARSCoV-2 S1 protein (MyBioSource, Cat\#MBS434277, San Diego, CA, USA). A goat anti-rabbit secondary antibody (IgG) conjugated with Alexa Fluor 594 (Invitrogen, Cat\#A-11037) or a goat anti-mouse secondary antibody (IgG) conjugated with Alexa Fluor 488 (Invitrogen, Cat\#A-11029) was used for visualization under a fluorescence microscope. For convalescent serum staining, cells were incubated with heat-inactivated serum and visualized with a goat anti-human secondary antibody (IgG) conjugated with FITC (Abcam, Cat\#ab97224, Cambridge, UK).

\subsection{Mouse Immunization}

Female C57BL $/ 6$ mice (7-9 weeks old, $n=3$ per group) were ordered from Nomura Siam International. Mice were given a prime-boost immunization intramuscularly (IM), spaced three weeks apart. For antigen formulation, SARS-CoV-2 S HexaPro protein (1 $\mu \mathrm{g}$ for the first dose and $5 \mu \mathrm{g}$ for the booster dose) was mixed with $100 \mu \mathrm{g}$ of aluminum hydroxide (Invivogen, Cat\#vac-alu-250). Serum was collected for analysis on study days 14,35 , and 56 after the initial immunization.

\subsection{Microneutralization Assay}

Heat-inactivated sera at $56^{\circ} \mathrm{C}$ for 30 min were two-fold serially diluted, starting with a dilution of 1:10. The serum dilutions were mixed with equal volumes of 100 TCID50 of SARS-CoV-2. After $1 \mathrm{~h}$ of incubation at $37^{\circ} \mathrm{C}, 100 \mu \mathrm{L}$ of the virus-serum mixture at each dilution was added in duplicate to Vero E6 cell monolayers in a 96-well microtiter plate. The last two columns are set as virus control, cell control, and virus back-titration. The plates were incubated at $37{ }^{\circ} \mathrm{C}$ in $5 \% \mathrm{CO}_{2}$ in a humidified incubator. After two days of incubation, the medium was discarded, and the cell monolayer was fixed with cold fixative (1:1 methanol:acetone) for $20 \mathrm{~min}$ on ice. Viral protein in the virus-infected cells was detected by ELISA assay. The cells were washed three times with PBST before blocking with $2 \%$ BSA for $1 \mathrm{~h}$ at room temperature. After washing, the viral nucleocapsid was detected using 1:5000 of SARS-CoV/SARS-CoV-2 Nucleocapsid monoclonal antibody (Sino Biological, Cat\#40143-R001) by incubation at $37^{\circ} \mathrm{C}$ for $1 \mathrm{~h}$. After removing the detection antibody, 1:2000 HRP-conjugated goat anti-rabbit polyclonal antibody (Dako, Denmark A/S, Cat\#P0448) was added, and the plate was incubated at $37^{\circ} \mathrm{C}$ for $1 \mathrm{~h}$. After washing, the TMB substrate (KPL, Cat\#5120-0075) was added. After 10 min incubation, the reaction was stopped by the addition of $1 \mathrm{~N} \mathrm{HCl}$. Optical density (O.D.) at 450 and $620 \mathrm{~nm}$ was measured by a microplate reader (Tecan Sunrise).

The virus neutralization endpoint titer of each serum was calculated using the following equation:

$X=[($ average A450 of virus control wells) - (average A450 of cell control wells) $] / 2+$ (average A450 of cell control wells)

The reciprocal of the highest dilution of serum with O.D. values less than $X$ is considered positive for neutralization activity. Serum samples that tested negative at a dilution of 1:10 were assigned an NT titer of $<10$. The serum that tests positive at 1:10 dilution will be reported as the NT titer of 20.

Each sample was carried out in duplicate. Live SARS-CoV-2 viruses at passage 3 or 4 and Vero E6 cells at the maximum passages of 20 were employed. The activities with live viruses were carried out in a certified biosafety level 3 facility. 


\section{Results}

\subsection{Expression and Purification of Recombinant SARS-CoV-2 HexaPro Spike Protein}

The prefusion-stabilized HexaPro construct (Figure 1A) encoding the spike ectodomain of SARS-CoV-2 with proline substitution at residues 817,892,899, 942,986, and 987, "GSAS" substitution at residues 682-685 (the furin cleavage site), and C-terminal foldon trimerization motif [14] was used to produce the HexaPro subunit vaccine in HEK293 cells. Transient transfection of HexaPro-encoding plasmid into the cells resulted in recombinant protein expression in the culture supernatant. The recombinant HexaPro protein was purified by Ni-NTA chromatography, followed by size-exclusion chromatography. The purity of the purified recombinant HexaPro was ascertained by SDS-PAGE (Figure 1B). Using pooled convalescence sera from COVID-19 patients and Western blot analysis, we show that the HexaPro spike is immunogenic.

A

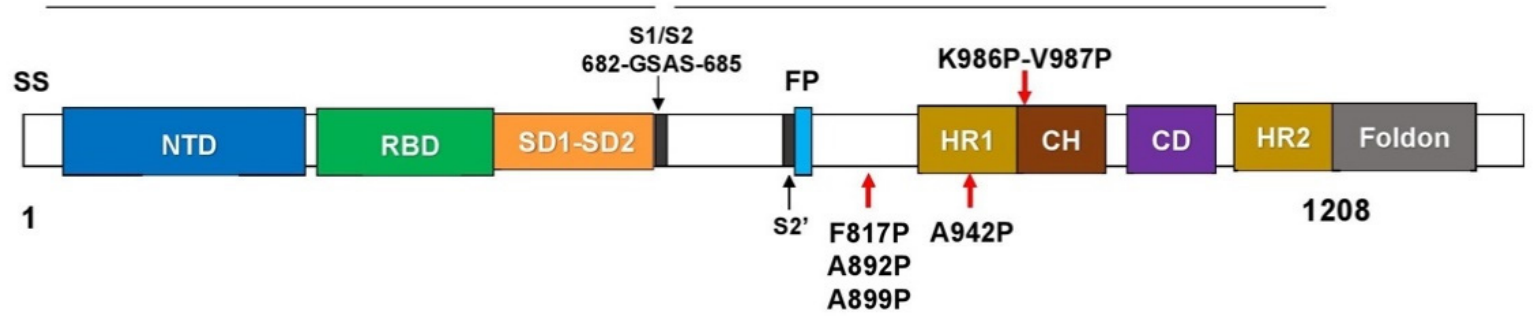

B

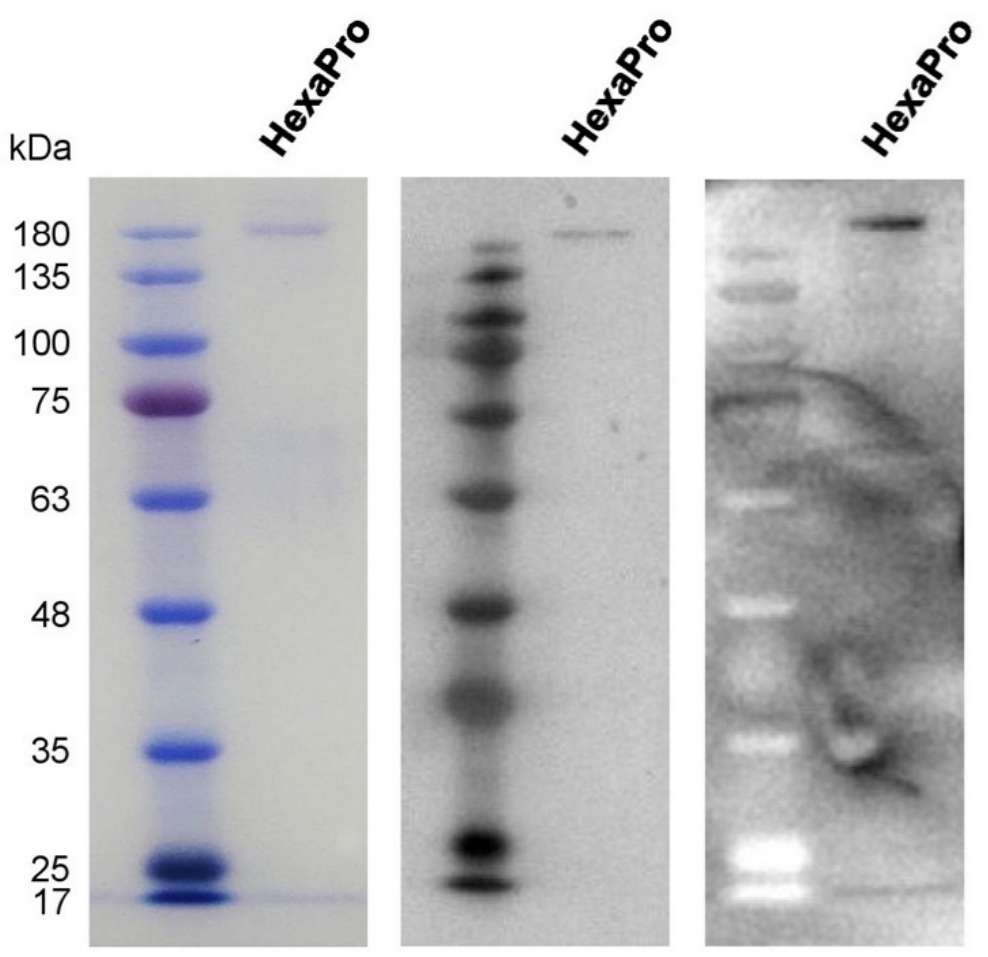

Figure 1. The recombinant SARS-CoV-2 HexaPro spike protein. (A) Schematic representation of the prefusion-stabilized SARS-CoV-2 HexaPro ectodomain showing the S1 and S2 subunits. Four additional proline substitutions from the S-2P construct are indicated by the red arrows shown below the construct. (B) The HexaPro protein expressed in HEK293T cells was purified and characterized by SDS-PAGE (left), Western blot using a commercial anti-RBD (middle), and Western blot using pooled convalescence sera (right). 
To confirm the immunogenicity of the HexaPro spike, immunofluorescence staining was then performed and this validated that antibodies could detect the spike structural variant against SARS-CoV-2 spike protein, which was also validated by the pooled convalescent sera (Figure 2). These results illustrate the potential of the HexaPro recombinant protein as a subunit vaccine.

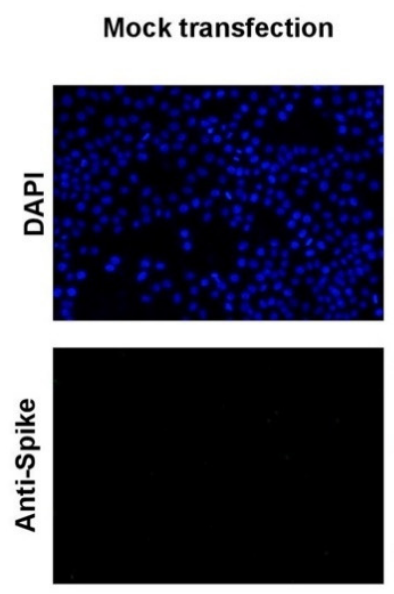

Patients' pooled sera
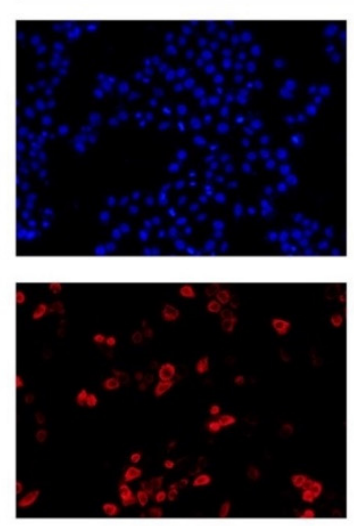

Anti-RBD
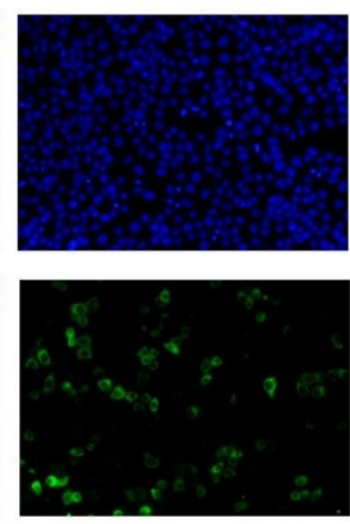

Anti-S1
HexaPro transfection
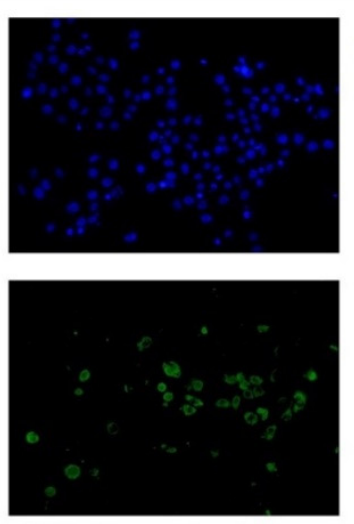

High-titer patient's sera
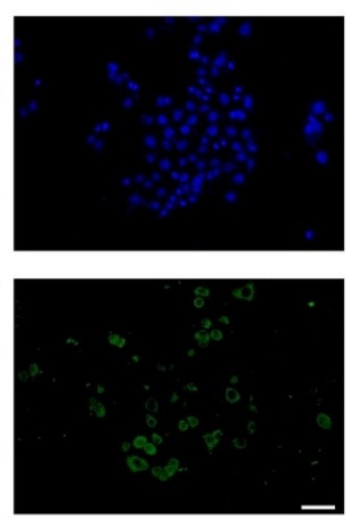

Patients' pooled sera

Figure 2. Immunofluorescence staining of the HexaPro spike expressed in HeLa cells using antibodies against spike RBD and S1 subunits, and convalescent sera derived from a patient with high-titer neutralization activity and from pooled sera. Scale bar: $50 \mu \mathrm{m}$.

\subsection{Neutralization of SARS-CoV-2 by Sera Collected from HexaPro-Immunized Mice}

The HexaPro subunit vaccine was then evaluated for its immunization activity via neutralization of SARS-CoV-2 by immunized mouse sera. An immunization protocol of low priming dose followed by high booster dose was followed. On day 0, C57BL/6 mice were prime-immunized with $1 \mu \mathrm{g}$ of HexaPro adjuvanted with aluminum hydroxide $(100 \mu \mathrm{g})$ via intramuscular administration. On day 21, the mice were boost-immunized with $5 \mu \mathrm{g}$ of HexaPro (Figure 3A). The microneutralization assay using live SARS-CoV-2 infection in Vero E6 cells was performed with sera collected on days 14, 35, and 56 after initial immunization. At 14 days after the priming dose, we did not observe a significant neutralizing activity in vaccinated mice (Figure 3B). However, sera from vaccinated mice collected 14 days after the booster dose elicit high neutralization titers. Furthermore, the level of neutralization activity was sustained at least 56 days after the initial immunization (Figure 3B). Together, our results indicate that the HexaPro subunit vaccine adjuvanted with aluminum hydroxide confers neutralization activity in sera collected from mice receiving the prime-boost regimen. 


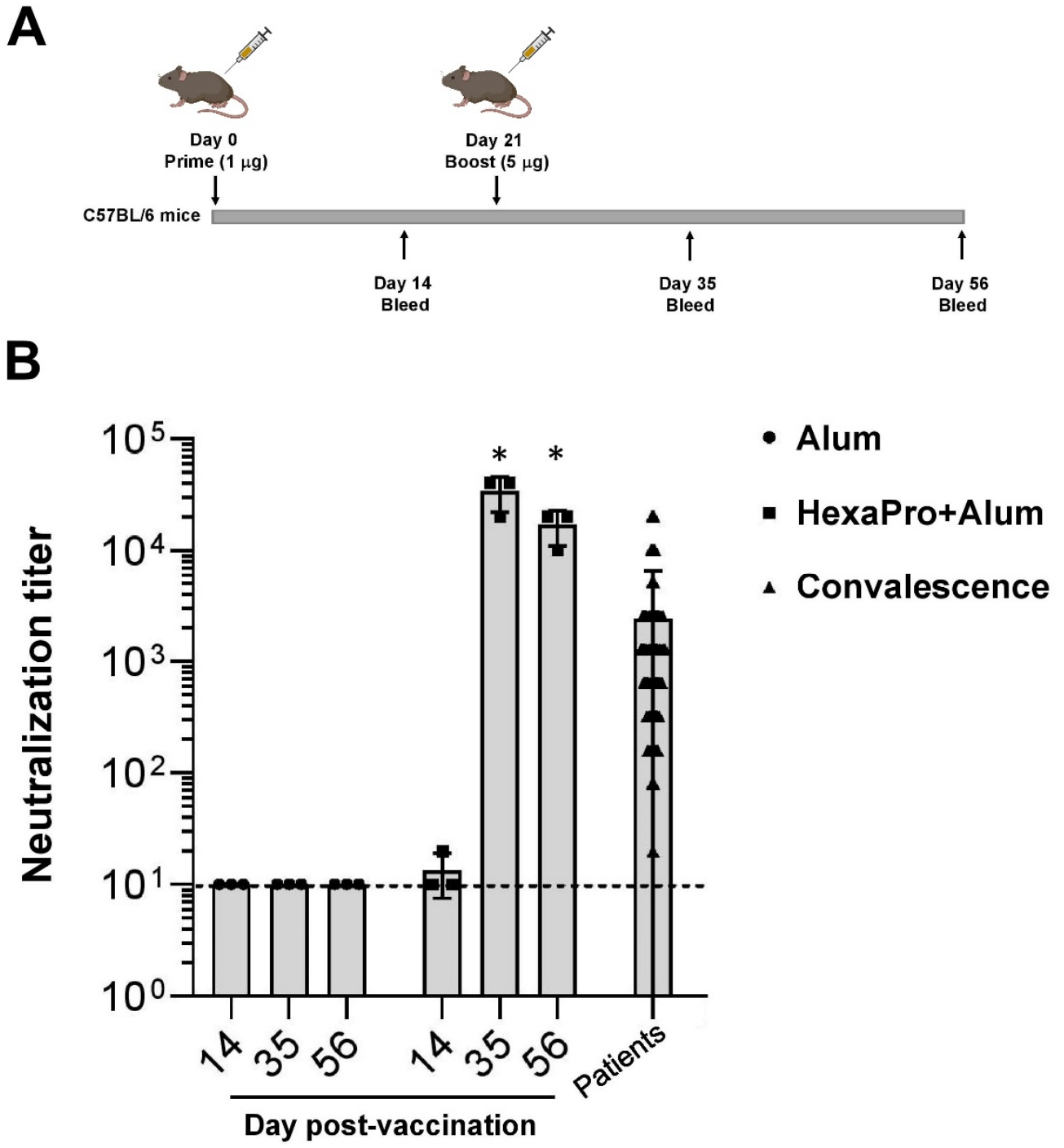

Figure 3. The prime-boost regimen using the recombinant HexaPro adjuvanted with aluminum hydroxide results in sera possessing neutralization activity. (A) C57BL/6 mice were vaccinated intramuscularly with Alum or HexaPro $(1 \mu \mathrm{g})+$ Alum. On day 21 they then received a booster dose with HexaPro $(5 \mu \mathrm{g})+$ Alum. (B) The virus neutralization endpoint titer of sera collected from mice and from convalescence sera. The dashed line shows the limit of detection. Neutralization activity on days 35 and 56 was compared to day 14. The error bars indicate the $\pm \mathrm{SD}$. Comparisons were performed using Student's $t$-test (unpaired, two tail); ${ }^{*} p<0.01$ (made in CBioRender-biorender.com (accessed on 2 March 2021)).

\section{Discussion}

In this report, we observed a potent SARS-CoV-2 neutralizing activity delivered by the subunit vaccine HexaPro spike; four amino acids of which were substituted by McLellan and colleagues into beneficial prolines leading to a more stable spike variant [14]. Specifically, the amino acid substitution was engineered within the S2 domain of the original S-2P spike [2]. This novel prefusion variant possesses $30 \%$ of the spike trimers being an "up" conformation with two exposed RBD, as opposed to just one exposed RBD found in S-2P. Due to its enhanced stability, the HexaPro spike has been proposed for its potential as a COVID-19 vaccine.

Using alum in vaccination has been shown to enhance activation of inflammatory dendritic cells and T-cell responses [15-17]. In a phase 1 trial, alum was employed with the inactivated SARS-CoV-2 vaccine BBV152 [18] as well as in ongoing clinical trials of COVID19 vaccines, including subunit vaccines (NCT04522089; NCT04527575; NCT04683484; 
NCT04742738) and an inactivated SARS-CoV-2 vaccine (NCT0464148). In addition, we also adopted a regimen of prime-boost immunization using a low priming dose followed by a high booster dose. A number of studies and clinical trials have demonstrated that a lower priming dose, followed by a subsequent higher booster dose, can induce more significant levels of the immune response [19-21], including the COVID-19 vaccine ChAdOx1 [13]. Importantly, effector cells are adversely induced by higher doses of antigen at prime immunization. On the other hand, immune memory cells are promisingly induced by lower doses at prime immunization, making this regimen suitable for long-term immunological memory [22].

Prefusion-stabilized spike proteins have been reported to facilitate their ectopic expression possibly by avoiding transition into a postfusion structure. Specifically, the HexaPro is more stable than its S-2P counterpart, which is difficult to be ectopically expressed and produced in mammalian cells [14]. Moreover, using biophysical assays, HexaPro has been shown to be more resistant to cold-induced denaturation than the previous generation S-2P variant [23]. Altogether, due to its highly stable conformation and feasible production, this HexaPro spike is potentially logistically applicable, and should be further developed into a COVID-19 vaccine and exploited for its efficacy in viral challenge studies using different SARS-CoV-2 genetic variants. Moreover, this HexaPro variant is suitable for development of next-generation mRNA, DNA, and viral vector vaccines. Indeed, an ongoing phase I/II clinical trial has already been initiated by the Government Pharmaceutical Organization of Thailand and Mahidol University for a viral vector vaccine, NDV-HXP-S, which utilizes the HexaPro spike as the SARS-CoV-2 antigen (NCT04764422).

\section{Conclusions}

We provide a proof-of-concept which indicates that the HexaPro subunit vaccine confers neutralization activity in sera collected from mice receiving the prime-boost regimen. We support the use of this HexaPro spike variant for next-generation COVID-19 vaccines.

Author Contributions: Conceptualization, A.T., S.H., P.W.; methodology, C.S., N.B., K.B., A.T., S.H., P.W.; formal analysis, C.S., N.B., K.B., K.S., S.M., S.L., N.P., C.P., N.K., P.W.; resources, S.C., A.T., S.H., P.W.; data curation, C.S., S.M., A.T., P.W.; writing—original draft preparation, C.S., A.T., P.W.; writing-review and editing, N.B., K.B., K.S., S.M., S.L., N.P., C.P., N.K., S.S. (Sirawat Srichatrapimuk), S.K., S.S. (Somnuek Sungkanuparph), S.C., S.H.; visualization, C.S., A.T., P.W.; supervision, A.T., S.H., P.W.; funding acquisition, S.C., P.W. All authors have read and agreed to the published version of the manuscript.

Funding: This research project was supported by the Faculty of Medicine Ramathibodi Hospital, Mahidol University, and CIF Grant, Faculty of Science, Mahidol University. C.S., S.L., and C.P. were supported by the Science Achievement Scholarship of Thailand. P.W. was supported by Mahidol University (New Discovery and Frontier Research Grant; grant number NDFR 11/2563) and the Office of National Higher Education Science Research and Innovation Policy Council by Program Management Unit for Human Resources and Institutional Development, Research and Innovation (PMU-B; grant number B05F630081). This research was funded by the Research Cluster Grant of Faculty of Medicine Ramathibodi Hospital, Mahidol University, grant number CF63010 (P.W. and S.C.). The APC was funded by Mahidol University.

Institutional Review Board Statement: Mouse experiments were performed under the Animal Ethics approved by Faculty of Science, Mahidol University (protocol code MUSC63-016-524; approved on 15 June 2020).

Informed Consent Statement: Not applicable.

Data Availability Statement: The data presented in this study is contained within the article.

Acknowledgments: This research project was supported by the Faculty of Medicine Ramathibodi Hospital, Mahidol University, and CIF Grant, Faculty of Science, Mahidol University. C.S., S.L., and C.P. were supported by the Science Achievement Scholarship of Thailand. P.W. was supported by Mahidol University (New Discovery and Frontier Research Grant; grant number NDFR 11/2563) and the Office of National Higher Education Science Research and Innovation Policy Council by 
Program Management Unit for Human Resources and Institutional Development, Research and Innovation (PMU-B; grant number B05F630081). This research was funded by the Research Cluster Grant of Faculty of Medicine Ramathibodi Hospital, Mahidol University, grant number CF63010 (P.W. and S.C.). The APC was funded by Mahidol University). Alum was kindly gifted by Waranyoo Phoolcharoen. We are indebted to COVID-19 Cluster and PW lab members for their suggestions and comments.

Conflicts of Interest: The authors declare no conflict of interest. The funders had no role in the design of the study; in the collection, analyses, or interpretation of data; in the writing of the manuscript, or in the decision to publish the results.

\section{References}

1. Hoffmann, M.; Kleine-Weber, H.; Schroeder, S.; Kruger, N.; Herrler, T.; Erichsen, S.; Schiergens, T.S.; Herrler, G.; Wu, N.H.; Nitsche, A.; et al. SARS-CoV-2 Cell Entry Depends on ACE2 and TMPRSS2 and Is Blocked by a Clinically Proven Protease Inhibitor. Cell 2020. [CrossRef]

2. Wrapp, D.; Wang, N.; Corbett, K.S.; Goldsmith, J.A.; Hsieh, C.L.; Abiona, O.; Graham, B.S.; McLellan, J.S. Cryo-EM structure of the 2019-nCoV spike in the prefusion conformation. Science 2020, 367, 1260-1263. [CrossRef] [PubMed]

3. Yan, R.; Zhang, Y.; Li, Y.; Xia, L.; Guo, Y.; Zhou, Q. Structural basis for the recognition of SARS-CoV-2 by full-length human ACE2. Science 2020, 367, 1444-1448. [CrossRef] [PubMed]

4. Walls, A.C.; Park, Y.J.; Tortorici, M.A.; Wall, A.; McGuire, A.T.; Veesler, D. Structure, Function, and Antigenicity of the SARS-CoV-2 Spike Glycoprotein. Cell 2020, 181, 281-292 e286. [CrossRef]

5. Ozono, S.; Zhang, Y.; Ode, H.; Sano, K.; Tan, T.S.; Imai, K.; Miyoshi, K.; Kishigami, S.; Ueno, T.; Iwatani, Y.; et al. SARS-CoV-2 D614G spike mutation increases entry efficiency with enhanced ACE2-binding affinity. Nat Commun 2021, 12, 848. [CrossRef] [PubMed]

6. Wang, P.; Liu, L.; Iketani, S.; Luo, Y.; Guo, Y.; Wang, M.; Yu, J.; Zhang, B.; Kwong, P.D.; Graham, B.S.; et al. Increased Resistance of SARS-CoV-2 Variants B.1.351 and B.1.1.7 to Antibody Neutralization. Nature 2021, 593, 130-135. [CrossRef] [PubMed]

7. Liu, Z.; VanBlargan, L.A.; Bloyet, L.M.; Rothlauf, P.W.; Chen, R.E.; Stumpf, S.; Zhao, H.; Errico, J.M.; Theel, E.S.; Liebeskind, M.J.; et al. Identification of SARS-CoV-2 spike mutations that attenuate monoclonal and serum antibody neutralization. Cell Host Microbe 2021, 29, 477-488. [CrossRef]

8. Wang, Z.; Schmidt, F.; Weisblum, Y.; Muecksch, F.; Barnes, C.O.; Finkin, S.; Schaefer-Babajew, D.; Cipolla, M.; Gaebler, C.; Lieberman, J.A.; et al. mRNA vaccine-elicited antibodies to SARS-CoV-2 and circulating variants. Nature 2021. [CrossRef]

9. Kirchdoerfer, R.N.; Cottrell, C.A.; Wang, N.; Pallesen, J.; Yassine, H.M.; Turner, H.L.; Corbett, K.S.; Graham, B.S.; McLellan, J.S.; Ward, A.B. Pre-fusion structure of a human coronavirus spike protein. Nature 2016, 531, 118-121. [CrossRef] [PubMed]

10. Pallesen, J.; Wang, N.; Corbett, K.S.; Wrapp, D.; Kirchdoerfer, R.N.; Turner, H.L.; Cottrell, C.A.; Becker, M.M.; Wang, L.; Shi, W.; et al. Immunogenicity and structures of a rationally designed prefusion MERS-CoV spike antigen. Proc. Natl. Acad. Sci. USA 2017, 114, E7348-E7357. [CrossRef]

11. Baden, L.R.; El Sahly, H.M.; Essink, B.; Kotloff, K.; Frey, S.; Novak, R.; Diemert, D.; Spector, S.A.; Rouphael, N.; Creech, C.B.; et al. Efficacy and Safety of the mRNA-1273 SARS-CoV-2 Vaccine. New Engl. J. Med. 2021, 384, 403-416. [CrossRef]

12. Polack, F.P.; Thomas, S.J.; Kitchin, N.; Absalon, J.; Gurtman, A.; Lockhart, S.; Perez, J.L.; Pérez Marc, G.; Moreira, E.D.; Zerbini, C.; et al. Safety and Efficacy of the BNT162b2 mRNA Covid-19 Vaccine. New Engl. J. Med. 2020, 383, 2603-2615. [CrossRef]

13. Voysey, M.; Clemens, S.A.C.; Madhi, S.A.; Weckx, L.Y.; Folegatti, P.M.; Aley, P.K.; Angus, B.; Baillie, V.L.; Barnabas, S.L.; Bhorat, Q.E.; et al. Safety and efficacy of the ChAdOx1 nCoV-19 vaccine (AZD1222) against SARS-CoV-2: An interim analysis of four randomised controlled trials in Brazil, South Africa, and the UK. Lancet 2021, 397, 99-111. [CrossRef]

14. Hsieh, C.L.; Goldsmith, J.A.; Schaub, J.M.; DiVenere, A.M.; Kuo, H.C.; Javanmardi, K.; Le, K.C.; Wrapp, D.; Lee, A.G.; Liu, Y.; et al. Structure-based design of prefusion-stabilized SARS-CoV-2 spikes. Science 2020, 369, 1501-1505. [CrossRef] [PubMed]

15. Kool, M.; Soullié, T.; van Nimwegen, M.; Willart, M.A.; Muskens, F.; Jung, S.; Hoogsteden, H.C.; Hammad, H.; Lambrecht, B.N. Alum adjuvant boosts adaptive immunity by inducing uric acid and activating inflammatory dendritic cells. J. Exp. Med. 2008, 205, 869-882. [CrossRef]

16. Kool, M.; Pétrilli, V.; De Smedt, T.; Rolaz, A.; Hammad, H.; van Nimwegen, M.; Bergen, I.M.; Castillo, R.; Lambrecht, B.N.; Tschopp, J. Cutting edge: Alum adjuvant stimulates inflammatory dendritic cells through activation of the NALP3 inflammasome. J. Immunol. 2008, 181, 3755-3759. [CrossRef] [PubMed]

17. HogenEsch, H.; O'Hagan, D.T.; Fox, C.B. Optimizing the utilization of aluminum adjuvants in vaccines: You might just get what you want. NPJ Vaccines 2018, 3, 51. [CrossRef]

18. Ella, R.; Vadrevu, K.M.; Jogdand, H.; Prasad, S.; Reddy, S.; Sarangi, V.; Ganneru, B.; Sapkal, G.; Yadav, P.; Abraham, P.; et al. Safety and immunogenicity of an inactivated SARS-CoV-2 vaccine, BBV152: A double-blind, randomised, phase 1 trial. Lancet Infect. Dis. 2021, 21, 637-646. [CrossRef]

19. Pace, D.; Khatami, A.; McKenna, J.; Campbell, D.; Attard-Montalto, S.; Birks, J.; Voysey, M.; White, C.; Finn, A.; Macloed, E.; et al. Immunogenicity of reduced dose priming schedules of serogroup $C$ meningococcal conjugate vaccine followed by booster at 12 months in infants: Open label randomised controlled trial. BMJ 2015, 350, h1554. [CrossRef] 
20. Nitayaphan, S.; Pitisuttithum, P.; Karnasuta, C.; Eamsila, C.; de Souza, M.; Morgan, P.; Polonis, V.; Benenson, M.; VanCott, T.; Ratto-Kim, S.; et al. Safety and immunogenicity of an HIV subtype B and E prime-boost vaccine combination in HIV-negative Thai adults. J. Infect. Dis. 2004, 190, 702-706. [CrossRef]

21. Sandström, E.; Nilsson, C.; Hejdeman, B.; Bråve, A.; Bratt, G.; Robb, M.; Cox, J.; Vancott, T.; Marovich, M.; Stout, R.; et al. Broad immunogenicity of a multigene, multiclade HIV-1 DNA vaccine boosted with heterologous HIV-1 recombinant modified vaccinia virus Ankara. J. Infect. Dis. 2008, 198, 1482-1490. [CrossRef]

22. Kardani, K.; Bolhassani, A.; Shahbazi, S. Prime-boost vaccine strategy against viral infections: Mechanisms and benefits. Vaccine 2016, 34, 413-423. [CrossRef] [PubMed]

23. Edwards, R.J.; Mansouri, K.; Stalls, V.; Manne, K.; Watts, B.; Parks, R.; Janowska, K.; Gobeil, S.M.C.; Kopp, M.; Li, D.; et al. Cold sensitivity of the SARS-CoV-2 spike ectodomain. Nat. Struct. Mol. Biol. 2021, 28, 128-131. [CrossRef] 